\title{
Juegos filosóficos, una apuesta hacia la educación del siglo XXI*
}

\author{
Erleysson Muñoz Gutiérrez \\ Paola Giraldo Tobón ${ }^{* * *}$
}

\begin{abstract}
Recibido: 18 de septiembre de 2014 - Revisado: 7 de noviembre de 2014 -
Aprobado: 12 de diciembre de 2014
\end{abstract}

\section{Resumen}

El juego filosófico se piensa como una apuesta que aborda el juego y la filosofía. Es un posible encuentro para potencializar el pensamiento crítico y creativo en los niños desde su ser-niño. Para llevar a cabo dicha propuesta, se indaga en las metodologías de filosofía para niños, así como la filosofía con niños abstrayendo de ambas partes las siguientes propuestas: comunidades de indagación, diálogo filosófico, pensamiento complejo y una experiencia impredecible de pensamiento filosófico que busca que el juego del niño atraviese las puertas de la filosofía -como práctica- con todo lo que es él, donde su percepción individual de lo que es la realidad pueda ser escuchada y hablada a través de la acción lúdica. Porque no hay nada más concreto que el Ser jugado.

Palabras clave: Juego, filosofía, niño, pensamiento complejo, diálogo.

* $\quad$ Artículo de reflexión, que constituye un avance del proyecto de grado en la Licenciatura en Filosofía de la Fundación Universitaria Luis Amigó titulado "El pensamiento filosófico en el aula de la educación básica primaria", adelantado por los autores de este trabajo.

** Estudiante de la Licenciatura en Filosofía de la Fundación Universitaria Luis Amigó. Dirección Postal: Transversal 51A 67B-90, Medellín, Colombia. Correo electrónico: erleysson@hotmail.com

*** Estudiante de la Licenciatura en Filosofía de la Fundación Universitaria Luis Amigó. Dirección Postal: Transversal 51A 67B-90, Medellín, Colombia. Correo electrónico: clarodelunaoscuro96@gmail.com 


\title{
Philosophical games, heading towards education of the 21 st Century*
}

\author{
Erleysson Muñoz Gutiérrez \\ Paola Giraldo Tobón***
}

\section{A bstract}

The philosophical game is intended as an approach that addresses both game and philosophy. It is an approach that aims to maximize the critical and creative thought in children from their "being-child". This paper explores the methodologies of philosophy for children, and philosophy with children abstracting from both the following proposals: communities of inquiry, philosophical dialogue, complex thinking and an unpredictable experience of philosophical thinking, which aims that children, through game, cross the doors of philosophy -as practicum-. As an experience where its individual perception of what reality is can be heard and talked about through playful activities. Because there is nothing more concrete than Being played.

Keywords: Game, Philosophy, Child, Complex thinking, Dialogue.

* Reflection article, which is an advanced development project in the Bachelor degree in philosophy from the University Foundation Luis Amigo entitled "The philosophical thought in the classroom basic primary education", advanced by the authors of this work.

** Student of the Bachelor of Philosophy of the University Foundation Luis Amigo. Postal Address: Transversal 51A67B-90, Medellin, Colombia. Email: erleysson@hotmail.com

*** Student of the Bachelor of Philosophy of the University Foundation Luis Amigo. Postal Address: Transversal 51A67B-90 Medellin, Colombia. Email: clarodelunaoscuro96@gmail.com 


\title{
Jeux philosophiques, un pari sur l'éducation du XXI siècle*
}

\author{
Erleysson Muñoz Gutiérrez \\ Paola Giraldo Tobón ${ }^{* * *}$
}

\section{Résumé}

Le jeu philosophique est pensé comme un pari qui aborde le jeu et la philosophie. C'est une rencontre possible pour potentialiser la pensée critique et créative des enfants depuis son être-enfant. Pour mener à bon terme cette proposition, des recherches sont faites à propos des méthodologies de philosophie pour enfants, ainsi que la philosophie avec des enfants, d'où sont abstraites les propositions suivantes : communautés de recherche, dialogue philosophique, pensée complexe, et une expérience imprévisible de la pensée philosophique qui cherche que le jeu de l'enfant traverse les portes de la philosophie -en tant que pratique- avec tout ce qu'il est, où la perception individuelle de la réalité puisse être écoutée et parlée par le biais de l'action ludique. Parce qu'il n'y a rien de plus concret que le fait d'Être joué.

Mots Clés: Jeu, philosophie, enfant, pensée complexe, dialogue.

L'article de réflexion, qui est un projet de développement de pointe dans le baccalauréat en philosophie de l'Université de la Fondation Luis Amigo intitulé «La pensée philosophique en classe éducation primaire de base", avancé par les auteurs de ce travail.

** Étudiants du programme de baccalauréat de philosophie de l'Université de la Fondation Luis Amigo. Adresse postale: Transversale 51A 67B-90, Medellin, en Colombie. Email: erleysson@hotmail.com

*** Étudiants du programme de baccalauréat de philosophie de l'Université de la Fondation Luis Amigo. Adresse postale: Transversale 51A 67B-90, Medellin, en Colombie. Email: clarodelunaoscuro96@gmail.com 


\section{Introducción}

En el plano educativo colombiano se presentan ciertas problemáticas que convocan a ser pensadas. Una de ellas -la más importante- se registra como un problema complejo y esencial. Una pregunta que preocupa, porque se instaura completamente en el mundo de la escuela y desde que se formuló hace tres décadas, aún no pierde vigencia y continúa siendo en el tiempo y en el espacio un interrogante por resolver: ¿Por qué la educación no enseña a pensar? Zuleta (2010) dirá: "la educación tal como existe actualmente, reprime el pensamiento, transmite datos, conocimientos, saberes y resultados que otros pensaron, pero no enseña ni permite pensar" (p. 11). Por lo que, pensar la educación hoy, significa reconocer que se enseña sin filosofía, el mayor cataclismo de la educación; donde se forma al niño en la disciplina desafortunada del hacer en una educación que tiende a producir mentalidades heterónomas, cuya causa ha sido una educación incapaz de provocar el deseo y la necesidad de aprender por parte de los niños. Ante esta prolongada crisis educativa, lo que se propone es hablar de filosofía, de los niños y de un posible encuentro entre ambos de la mano de maestros comprometidos con el conocimiento y la aventura de educar.

La idea de una filosofía en la escuela se piensa para potencializar las habilidades cognitivas y de razonamiento en los niños y las niñas. Fundada en el interés de los mismos pequeños, la autonomía desde la escucha y el diálogo consigo mismo y con otros, y juegos filosóficos como una apuesta hacia la educación del siglo XXI; holística, democrática, dialógica, filosófica, investigativa. En otras palabras, filosofía pensada desde lo complejo para cultivar espíritus curiosos, críticos y creativos; porque la escuela debe ser capaz de brindar a niños y niñas las herramientas adecuadas en el momento en que empiezan a interrogarse por el mundo y su inserción en el mismo. Puesto que, una autentica educación no puede no ser filosófica -al ser ésta el único saber que enseña a vivir-. De este modo, no es cualquier filosofía la que importa practicar con los niños.

Las teorías que aportan en la realización del proyecto, contemplan aspectos filosóficos, políticos, pedagógicos, científicos, formando un tejido que compromete a la totalidad de las áreas del saber. La metodología de la propuesta transita en el programa de filosofía para niños y el enfoque de filosofía con niños que se estudian para hacer práctico el proyecto. Todo en la consecución por un conocimiento integrador, no excluyente y no fragmentado, que pueda estar al alcance de los pequeños a la hora de cuestionar y entender el contexto y la realidad de la cual hacen parte. Esta es la apuesta por una educación pensada que permita y enseñe a pensar.

Hace más de tres décadas que el buen pensador Estanislao Zuleta (2010) develó el problema central de la educación: en la escuela se enseña sin filosofía y en ninguna de las asignaturas escolares la filosofía está presente, inclusive recalca la ausencia de filosofía en la misma filosofía. La educación por tanto, se ancló en enseñar resultados sin enseñar los procesos del conocimiento, produciendo 
individuos heterónomos con un mínimo de autonomía y con el máximo de dependencia hacia los demás. A su vez, afirmó que en este país los maestros llevan años haciéndoles el mal favor a los estudiantes de ahorrarles la angustiosa tarea de pensar y de conocer.

Ahora ante el interrogante del por qué se cree que el sistema educativo colombiano requiere ser intervenido por pedagogías formadoras, la respuesta no se da más que por la necesidad presente en las instituciones educativas de repensar la forma en la que está llevando a cabo el proceso de educar a maestros, niños y jóvenes en la aventura del conocimiento.

Bien se dice que "una educación sin filosofía es vacía y una filosofía sin educación es desierta" (Noro, 2013, p. 23). Es por eso que el buen Estanislao Zuleta pensaba que la educación debería tener como suelo epistemológico la filosofía, para darle un sentido a la escuela al momento de educar, para que se atreviera a pensar y a dejar pensar, para que se aventurara en los diferentes discursos de las variadas disciplinas; porque más que transmitir corrientes del pensamiento, la filosofía es un saber que enseña a vivir y a pensar de forma crítica y reflexiva haciendo lecturas y relecturas del mundo. El mismo Montaigne, pensador del renacimiento se cuestionaba al respecto, ¿Por qué no dejar que los niños aprendan las cosas de la vida a través de la filosofía?

Pero la idea no es caer en un romanticismo, sino en indagar ¿Cómo lograr llevar la filosofía al aula de tal forma que el niño haga las veces de protagonista de su conocimiento? ¿Qué métodos serían los más apropiados para proceder? Una posible propuesta surgió desde juegos filosóficos cuyos fundamentos teóricos se encuentran anclados en las metodologías de filosofía para niños y filosofía con niños. Una apuesta que se espera resulte innovadora en sí misma y que pueda beneficiar el mundo de la escuela desde otra perspectiva, al tiempo que sensibilice a los actores que intervienen en ella para que se arriesguen a pensar nuevas formas de intervenir el campo de la educación del siglo XXI.

Para saber cómo se pensó la apuesta por juegos filosóficos, primero se tratará a grosso modo qué se entiende por filosofía para niños y cómo se desarrolla el programa, lo mismo se hará con el enfoque de filosofía con niños; destacando de ambas partes los aportes que se tuvieron en cuenta para la construcción teórico-práctica de los juegos a la vez que dé soporte para entrar a problematizar el juego en el ámbito educativo.

\section{Filosofía para niños}

El programa de filosofía para niños $(\mathrm{FpN})$ aparece en Estados Unidos a finales de los años sesenta, con el profesor Mathew Lipman de la Universidad de Montclair en New Jersey junto con Ann Margaret Sharp. La idea del programa parte de la experiencia del profesor en sus clases de lógica con los estudiantes 
que presentaban dificultades en habilidades de pensamiento y ámbito cognitivo. Lipman en su obra filosofía en el Aula (1992) determina que el problema adviene de la escuela por su tendencia a enseñar memorísticamente negando la promoción del pensamiento. Es necesario crear una educación con un enfoque heterogéneo, que ayude y anime al estudiante a pensar por sí mismo, liberándolo de hábitos mentales que no son críticos ni cuestionables, se busca así incentivar en el niño un pensamiento o capacidades críticas y reflexivas ante su contexto. Esta apuesta es controvertible ¿Por qué la filosofía? ¿Cuáles son las facultades que se utilizarán de esa disciplina? ¿Cómo la llevará a la práctica? Para desarrollar dichas destrezas anteriormente expuestas, la filosofía brindará las técnicas para razonar, investigar, realizar un aprendizaje conceptual, también se postula como una forma de vida.

Según Lipman (1992), se trata de convertir el aula tradicional en una comunidad de diálogo o de investigación conjunta, participativa y cooperativa, en la que alumnos y profesores busquen conjuntamente las respuestas a cuestiones planteadas. El programa de FpN consta de siete novelas, cada una tiene una problemática social o cognitiva diferente, acompañada de un manual para que el docente pueda trabajar el concepto expuesto o las diferentes dificultades que presenta el texto. El método es guiado por las comunidades de investigación que partirán de la pregunta y el diálogo que se pueda generar en esta intervención en el aula. Las novelas que se utilizan en este programa se integran al currículum, como obras de ficción "que extraen por sí mismo, las leyes del razonamiento y descubren puntos de vista filosóficos alternativos" (Carmano, 2005, p. 115). Las edades que se emplean en estas novelas son: 5 a 9 años con un enfoque hacia el desarrollo de conceptos e investigación, de 10 a 13 están más preparados para entender los principios de razonamiento, 14 a 17 años utilizan los principios de razonamientos teóricos y prácticos en el contexto escolar y personal. Este programa se apoya en varios pilares; comunidad de investigación, diálogo y pensamiento complejo, esto no significa que no tenga más dimensiones para ocuparse.

A continuación se expone uno de los métodos que se utiliza de manera más intensiva en el juego filosófico, según Peirce (1987), Kuhn (1993), Dewey (1973) y Lipman (1997) estas son las comunidades de indagación. El concepto de comunidad que implementa Lipman lo construye desde diferentes puntos de vista, el primero es Peirce (1987) no existe una comunidad científica neutral porque todo depende de la relacón de los científicos. Continuando con la expresión de comunidad científica están las ideas de Kuhn (1993) que tiene el mérito de haber puesto de "relieve el carácter sociológico del desarrollo científico y de sus interrelaciones con otros aspectos de la cultura" (Carmano, 2005, p. 108). La noción de comunidad expuesta por Kuhn se basa en el trabajo en equipo de los científicos para cuestionar, construir y resolver los enigmas, a partir de los diferentes campos de la investigación. Esta visión de la comunidad científica se integra a la educación con Dewey (1973) desde el ámbito pedagógico: el aula como una pequeña comunidad. Por último Lipman (1992) el cual expresa que la comunidad de investigación tiene sus propiedades en la filosofía y el diálogo. 
La relación entre Dewey y Lipman se desarrolla por considerar que es posible el crecimiento personal cuando el individuo entra en proceso de interrelación con otro. Se preocupan por una misma fundamentación pedagógica considerando al niño como un ciudadano en toda la palabra. La diferencia entre ellos inicia con Dewey considerando que al niño no le interesa la abstracción y racionalización, en cambio Lipman asegura que es capaz de llegar a ella. Pera ambos el pensamiento reflexivo se fundamenta en el proceso educativo; en Dewey es la ciencia y Lipman la investigación filosófica, en esta última, se produce o encuentra el proceso cognitivo a través del ejercicio dialógico (razonamiento) apuntando a la formulación de juicios, desarrollo de habilidades y destrezas del pensamiento evitando adoctrinamientos por medio de la reflexión de valores, creencias, normas y actitudes.

El ideal con las comunidades de indagación aplicada a la estructura del juego es lograr que el maestro tenga una visión holística en los niños y jóvenes, porque ellos tienen una capacidad de comprender racionalmente los interrogantes que afrontan cotidianamente. Este objetivo se cumple en dos enfoques: primero, la investigación ética cumple la función de identificación y comprensión de los diversos valores, logrando que las personas vayan desarrollando alguna razonabilidad. En segundo lugar está la educación moral que implica la formación de los hábitos que conforma nuestro carácter desde el nacimiento. El ideal de una educación moral es llevar a los niños al desarrollo de sus propios hábitos, indagando sobre estos, de esta manera la educación moral incluye la investigación ética como uno de sus factores esenciales. Los niños no solo se pueden limitar a ser agentes morales, necesitan desarrollar por sí mismos la capacidad de gestionar adecuadamente cada situación que se les presente; la persona ética aprenderá a actuar en comunidad, a compartir, intercambiar y someter los conceptos morales a juicios rigurosos sin enfoques psicológicos o terapéuticos.

\section{El diálogo}

El diálogo socrático es el modelo que orienta las comunidades de investigación posibilitando el debate, las soluciones a los problemas y hacer asumible aquello que ha sido objeto de indagación. Orientado por criterios lógicos que potencializa a sujetos críticos y creativos en el aula con las comunidades de investigación. La curiosidad por el conocimiento parte de la pregunta, del deseo de encontrar una respuesta verdadera, para ello Lipman recurre al diálogo como ejercicio de interacción entre la pregunta y respuesta generada en el entorno del discente. Salazar (1967) afirma:

Cuando se está aplicando el método del diálogo, se está direccionando el aprendizaje, se desenvuelve mediante un encadenamiento de interrogaciones y respuestas, gracias al cual el pensamiento del educando es incorporado por la acción del profesor, en el proceso dialéctico de la reflexión filosófica (p. 50). 
La noción del diálogo no es tomado como un método sino un procedimiento, táctica o estrategia. El trabajo del maestro será incentivar en sus estudiantes el ejercicio del preguntar. Cuando se logra esta intervención del profesor hacia el estudiante se genera una participación constante y activa en el aula.

Para un trabajo pedagógico y epistemológico el aula se piensa como una comunidad universal de investigadores con el ideal de una producción, reconstrucción social, desarrollo afectivo, político, cultural, ético de los discentes y docentes. Convertir el juego en un tipo de comunidad de indagación es fortalecer en los estudiantes la escucha de unos a otros con respeto, construyendo sus propias ideas teniendo en cuenta las expuestas, es un ejercicio que brinda la posibilidad de reforzar argumentos no muy bien fundamentados.

Desarrollar esta práctica dialógica con un enfoque reflexivo es un proceso de reconstrucción de la experiencia. En consecuencia, según Sharp y Splitter (1996) el niño por determinadas didácticas implementadas potencializará el diálogo argumentativo que posibilitará, entre otras capacidades, corregirnos cooperativamente, detectará las falacias, inferencias, manifestará supuestos, establecerá analogías, proporcionará buenas razones, generará hipótesis, criterios, formará conceptos.

Generar un diálogo con las características mencionadas anteriormente, es afirmar la propuesta de filosofía para niños desde sus novelas y manuales que se implementa al currículum, pero este tipo de pedagogía establece limitaciones en el pensamiento del discente y el aula. “La experiencia de pensar filosóficamente se confunde así con un ejercicio de aplicación de reglas lógicas, se transforma en habilidades mecánicas, justamente por pensar que el sentido es apenas una cuestión de articulación lógica" (López, 2004, p. 121). Este tipo de aprendizaje no será factible para todos los discentes.

La idea que los niños se relacionen con el pensamiento filosófico no fue concebida por Lipman, anteriormente diferentes filósofos ya lo habían sugerido. Sin embargo, este profesor estructuró una propuesta teórica práctica para llevar a cabo la filosofía en el aula. En su propuesta metodológica (las novelas) los protagonistas son niños que tienen situaciones y circunstancias cotidianas que se relacionan con la vida real del lector norteamericano; a pesar de las traducciones de sus textos al español, sus novelas no se adaptan a una perspectiva de América Latina, en cambio, una visión más integra de la filosofía en educación la posibilita Walter Kohan en Brasil desde filosofía con niños ( $\mathrm{FcN})$.

Esta perspectiva permite al estudiante desenvolverse de manera libre, espontánea y creativa a partir del juego porque ofrece dimensiones subjetivadas, prácticas y objetivas; por eso $\mathrm{FcN}$ permite una diversidad de paradigmas dentro de su definición, componente y fundamentación. 


\section{Filosofía con niños}

Filosofía con niños es vivir e indagar en la experiencia, es una constante búsqueda de respuestas antes los problemas no resueltos. La filosofía en escuela como práctica hace que los niños intervengan en ella, que ambos términos (filosofía y niño) se modifiquen en su encuentro, que sea accesible y pertinente. Se presenta un desafío en lo teórico y metodológico: el primero se fundamenta en el programa de Lipman con la relación de filosofía y educación que se tornan indispensables, porque sin filosofía la educación sería vacía y ciega, ambas son prácticas históricas y sociales por los aportes que le brinda al hombre en su proceso de enseñanza/aprendizaje. Sin embargo, la filosofía no puede seguir limitada en sus bases teóricas, hay que realizar una abstracción para llevarla a pensar la praxis. Las instituciones escolares no tienen una visión histórica fuera de las actuales sociedades neoliberales, es necesario que incorporen interpretaciones como la del marxismo, el post-estructuralismo y la teoría crítica para obtener un panorama más amplio de la función social de la educación.

Lipman desarrolla una caracterización importante hacia el niño considerándolo un ser crítico, creativo, curioso, lúdico, racional y con un posible desarrollo del diálogo filosófico. Esto no es una visión de un adulto pequeño, para no caer en este error hay que escucharlo y preguntarle ¿Cuál es la visión de sí mismo? Solo con esto no bastará porque tradicionalmente la psicología evolutiva o psicopedagogía creen saber todo del niño, olvidando el ejercicio de estar constantemente debatiendo por la pregunta ¿Qué es un niño? Se piensa que ya está resuelta.

El segundo desafío lo constituye la metodología a partir del rol que ejerce el maestro en la enseñanza de la filosofía, considerando al estudiante como una tabla rasa, olvidando lo significativo que es la experiencia o conocimiento que este pueda traer en su proceso de aprendizaje.

Para realizar una apuesta de FpN y FcN en el juego es necesaria la evaluación en los conceptos de enseñanza/aprendizaje, es importante porque enseñar filosofía no siempre tiene que ser desde lo teórico sino también desde lo práctico, se debe tener en cuenta que su enseñanza no se puede encasillar en métodos o programas, por el contrario, podría posibilitar una didáctica en la cual se construyan conjuntamente todos los saberes entre los agentes internos y externos de la institución.

¿Por qué la importancia de la filosofía en el aula? Esta será constituida desde una interdisciplinariedad/transdisciplinariedad/multidisciplinariedad que se implemente en edades de correspondientes aprendizajes constructivos, donde el diálogo que se conciba no sea una obstrucción al pensamiento individual de cada niño dependiente de su historia, sino, considerando el texto, contexto, meta-contexto que surja del sujeto, se realice una transculturalidad, haciendo posible una sensibilidad desde la música, literatura, teatro, cine, artes plásticas y una sensibilidad ante los problemas de ese estudio. 
Para generar este cambio en la educación del siglo XXI el educador necesita tener una perspectiva holística sobre su quehacer como docente, cuando se logra esta visión comienza a revaluarse la concepción de evaluación. Así, es necesario un evaluador que forme o posibilite los instrumentos para poder trabajar en una filosofía práctica, crítica y reflexiva con los estudiantes. El motivo de este análisis surge por el pensamiento tradicional implementado al docente, dificultando las nuevas propuestas pedagógicas, la asimilación de una evaluación cualitativa y formativa. Considerar la evaluación como un acuerdo de rendimiento del discente es excluir a aquellos que revelan dificultades en el desarrollo de las competencias necesarias, en cambio si utilizamos adecuadamente los instrumentos de la evaluación ésta se tornará una actividad cualitativa y valorativa. Como expresa Solé (1993) en la actividad formativa, es preciso que, dentro de su carácter pedagógico, se adopte uno regulativo para la enseñanza/aprendizaje. El profesor necesitará reformular y repensar la evaluación constantemente teniendo en cuenta las necesidades de sus alumnos, de este modo, la evaluación servirá como instrumento que orientará la enseñanza, donde se tome conciencia de lo aprendido. Para alcanzar este método es necesario, en primer lugar, posibilitarle al estudiante una autoevaluación, en segundo lugar, valorizar al docente promoviendo un contexto propicio para la reflexión y superación de dificultades que se puedan presentar.

La labor del maestro en la evaluación necesitará estar orientada hacia: primero, identificar, definir indicadores y sistematizar las experiencias evaluativas. Segundo, tener en cuenta el procedimiento de un buen desempeño o mal desempeño, donde él mismo realice una autoevaluación de su trabajo y comprenda por qué se han equivocado sus alumnos proponiendo nuevas acciones para una solución considerando la importancia del trabajo en grupo. Los diferentes modos de evaluar según Jiménez, González y Hernández (2011) son: autoevaluación, coevaluación, heteroevaluación, por lo que se llevan a cabo registros diarios de observador para trabajar sobre las fortalezas y debilidades de cada estudiante, en función de su necesidad. Tercero, realizar un examen de conciencia sobre su actuación pedagógica es un proceso de aprendizaje personal o grupal teniendo en cuenta todo lo que realizó para aprender.

En cuanto al papel del alumno en la evaluación, creemos que podría cumplir las siguientes funciones: tomar conciencia de su proceso de aprendizaje, valoración, intervención, disposición al trabajo en equipo e individual, así como participar activamente en la revisión o análisis de los resultados. La evaluación necesita contener el proceso de enseñanza/aprendizaje porque la tarea de un agente evaluador es seguir investigando las diferentes maneras de educar y compartir el conocimiento. Al lograr este objetivo se identificarán las múltiples dificultades, que son necesarias superar, pero al mismo tiempo, debe entenderse como un mecanismo de reconocimiento e innovación constante del proceso pedagógico. Al postular el juego como una propuesta metodológica, que incluye la evaluación, se pretende darle un lugar práctico y teórico a la pedagogía, para que se incluya en el currículo. 
Combinar FpN y FcN desde un juego es potencializar en el estudiante la racionabilidad, experiencia, lo crítico y creativo para usarlo no solamente en la escuela sino fuera de ella. Se ha mencionado la racionalidad y la experiencia, pero lo crítico y creativo hace parte del pensamiento complejo que propone Mathew Lipman, esta es una de las apuestas de juegos filosóficos que busca generar el pensamiento crítico y creativo para llegar al de orden superior.

\section{Pensamiento complejo}

El planteamiento para desarrollar una nueva forma de pensamiento es postularlo desde una razonabilidad, un pensar desde la complejidad, reivindicándole al individuo una trasformación de toda realidad. Para ser razonable es necesario explicar la relación que existe entre la expresión "razón" y "racionalidad"Como afirma Ruano (1996) esta última no hace sino referencia a un estado, a una manera estática de la razón, además, lo racional es simplemente aquello que está dotado de razón.

La racionalidad se entiende "como ordenación o sistematización básicamente metodológica, es decir, como referencia a una razón pura, formal y objetiva de tipo matemático, cuya manifestación es una forma de razonar apodíctica que se fundamenta en la demostración y concluye en premisas verdaderas o falsas" (Cuno, 2010, p. 215).

La razonabilidad, en cambio, depende de la noción de aceptación, la misma que fue introducida por el propio Perelman (1997) cuya idea central se encuentra en la sustitución del requisito "prueba de racionalidad en proposiciones valorativas" cambiando a la "aceptación", que conduce a no pretender prioritariamente encontrar una verdad demostrable, sino más bien, aceptable, es decir, razonable. Atribuyéndole "un sentido moral, es decir, con la capacidad para concebir a los otros individuos como libres e iguales y entablar con ellos una relación de cooperación sobre bases equitativas" (Rivera, 1997, p. 54).

Lipman en su texto Pensamiento complejo y educación (1997) expresa que los jóvenes aprenden y crecen como seres razonables, teniendo en cuenta que la racionalidad lleva en su seno la irracionalidad. Quizá por eso plantea los límites y contradicciones de lo racional, pero es la única salida, por lo tanto propone una nueva racionalidad asentada sobre otras bases, desde un humanismo radical, crítico y problematizador.

Pensar en complejidad dirá Morin (1983) es un abordaje de las complicaciones, inseguridades, incertidumbres, contradicciones, es ir más allá de lo evidente, es construir desde lo latente, es un develar del otro y con lo otro, habitando constantemente la interacción del juego; sujeto, objeto, mundo, universo e individuo. 
Las dimensiones que plantean Edgar Morín y Matthew Lipman sobre el pensamiento complejo generan una pesadumbre en el mundo, en la educación e individuo. Morín lo expresa como "el tejido de eventos, acciones, interacciones, retroacciones, determinaciones, azares, que constituyen nuestro mundo fenoménico" (Morín, 2003, p. 32) y el segundo lo afirma como la función que tiene el individuo de ser "consciente de sus propios imaginarios e impedimentos; son razones y evidencias en las que se apoyan sus conclusiones, examinado su metodología, procedimientos, perspectivas y puntos de vistas propios. Educado para identificar los factores que llevan a la parcialidad, al prejuicio y autoengaño" (Lipman, 1997, p. 67). El desarrollo de un pensamiento complejo en Lipman es entendido como el pensamiento de orden superior, dado que este lleva a la complejidad. La preocupación de ambos autores es la del cómo el conocimiento se está aprendiendo y enseñando desde lo macro a lo micro y de lo micro a lo macro en los diferentes paradigmas.

El pensamiento complejo puede entenderse como "la disposición de apertura ante la crisis del paradigma de la simplificación y la reducción, que eventualmente podría convertirse en un nuevo paradigma, pero que no pretende autopredicarse como el nuevo paradigma alternativo" (Moreno, 2002, p. 21). El pensamiento complejo es una auto-organización de conocimiento que vincula lo desvinculado, conteniendo su antagonista, el pensamiento simplista, el cual establece que para poder conocer hay que separar el sujeto del objeto. La complejidad es ante todo una noción lógica, epistemológica y política, un pensamiento donde no se puede ser espectador, sino actor que piensa constructivamente. En palabras de Morín, lo más adecuado para introducirse en el pensamiento complejo es: "si quieres saber qué es el pensamiento complejo, empieza complejizando tu pensamiento" (Morín, 1983, p.29). El pensamiento complejo se desarrolla a condición de un autoconocimiento. De este modo modifica la frase socrática: "Conócete a ti mismo conociéndote" (Moreno, p.29).

Este pensamiento es enriquecido por tácticas meta-cognitivas, auto-correctivas y capacidades que lleve al pensamiento a una reflexión sobre su propia metodología en el contenido que se trabaja. Esto lleva a la cuestión del ¿Cómo desarrollar este tipo de pensamiento? Lo primero es definir el pensamiento de orden superior (P.O.S) y cuál es su aplicabilidad en este proceso, después, con esta misma metodología la concepción del pensamiento de orden inferior (P.O.I) por último qué relación tiene el P.O.S con las comunidades de investigación.

El P.O.S. "se conforma como el conjunto de las actividades mentales transformativas requeridas para el análisis de situaciones complejas y la emisión de juicios ponderados de acuerdo con múltiples criterios" (Resnick, 1987, p. 44). Un pensamiento de orden superior "no implica un diálogo de palabras, sino, uno entre estilos de pensamientos, métodos de análisis, perspectivas epistemológicas y metafísicas" (Lipman, 1997, p. 119). También incluye dimensiones emotivas, afectivas y sociales del pensar, por lo tanto se compone del pensamiento crítico y pensamiento creativo posibilitando el pensamiento de orden superior. 
Como se explicará, el pensamiento crítico está compuesto por el razonamiento, las implicaciones, la inferencia y la habilidad. El razonamiento se entiende como las operaciones simbólicas y la composición de acciones cognitivas que pone en marcha dichas operaciones. La implicación: “es una operación lógica mediante la cual, en un argumento válido, pueden afirmarse las premisas para implicar la conclusión" (Lipman, 1997, p. 126). La inferencia: "es una acción cognitiva en la que alguien extrae la conclusión de las premisas. Tanto las acciones como las funciones pueden realizarse correcta o incorrectamente, esto dependerá de los criterios o normas que podamos aplicarles" (Lipman, pp. 126-127). Por último, 'la habilidad' es la capacidad para organizar acciones y funciones que logren el efecto deseado. El pensamiento crítico en cuestiones de creencias asume un papel importante, el cual es resguardarnos de esas ideas convencionales que no se han reflexionado o investigado. Desarrollar este pensamiento en escuelas atiende a la necesidad de crear foros de libertad con condiciones académicas para los niños y niñas similares a los universitarios, posibilitando la formulación de juicios, especulaciones o hipótesis sin alguna barrera instaurada. La libertad de las ideas garantiza un P.O.S. Desafortunadamente la mayoría de las escuelas potencializan en los estudiantes "hábitos de inhibición intelectual" (Lipman, pp. 16-17).

El pensamiento creativo "es aquel que conduce al juicio, orientado por el contexto, autotrascendental y sensible a criterios. Efectivamente el pensamiento creativo es sensible al criterio de verdad, pero está regido por el contexto de la investigación al que tiene lugar. Y evidentemente el pensamiento crítico es sensible al contexto, pero los criterios de verdad, racionalidad y significado son prioritarios en éste" (Lipman, 1997, p. 265). Ambos pensamientos tienen su propio objetivo, el primero se interesa en una globalidad, en la invención y en trascender (ir más allá de sí mismo); lo analítico no lo contienen pero no es del todo indiferente, se aplica como un proceso y no un resultado. El segundo "mantiene un interés primordial por la verdad, entonces, tiene una preocupación auténtica por evitar el error y la falsedad" (Lipman, p. 267).

En la investigación el pensamiento crítico y creativo son flexibles en las determinadas situaciones que presentan indagación. El pensamiento crítico a través de los criterios y conceptos aporta al proceso, en cambio, el creativo es "sensible al modo en que la cualidad pervasiva se encarna de valores, significados y esquemas potentes que buscan orientar su pensamiento en una dirección u otra" (Lipman, 1997, p. 266). Los dos pensamientos contienen el rasgo de la sensibilidad al contexto, aunque en el creativo es más importante por su influencia que el crítico, por lo tanto son muy diferentes. Otra de sus incompatibilidades es el modo de emplear los criterios: en el pensamiento creativo "los criterios tienden a componerse a través de pares relacionados dialécticamente o sistemas en los que cada concepto se sobrepone uno a otro de forma opuesta y donde la tensión entre estos conceptos ayuda a crear esta fuerza mágica que subsiste en todo trabajo creativo" (Lipman, p. 281). 
La pregunta por la complejidad en la escuela es también la pregunta por la permanencia del hombre en el mundo, el pensamiento complejo incluye dimensiones emotivas, afectivas y sociales del pensar. Las escuelas deberían iniciar la formación de personas que razonen adecuadamente, realizando buenos juicios y que se pueden abrir a diversos e innovadores pensamientos, ya que esta es la finalidad de la educación. El pensamiento de orden superior es el que posibilita lo anterior expuesto, recordando que los diferentes procesos o niveles varían en los niños. Para estimular este pensamiento se invita a que los estudiantes hagan filosofía utilizando la comunidad de investigación, este procedimiento no es un limitante, la idea es interactuar con todo tipo de metodología en el aula, para poder provocar discusión y reflexión sobre la materia de cualquier área de conocimiento. Además utilizar el pensamiento crítico unido al creativo es incitar a cualquier debate académico, disciplinario y artístico.

Hay aspectos de la vida humana que no se pueden resolver con la misma precisión de un método científico, es necesario analizar algunas acciones humanas, porque no hay correspondencia entre el pensamiento y conducta. La satisfacción que brindan las metas razonables o sensibles aun cuando no sean del todo racionales, se refleja en las discusiones éticas porque en algunos casos no se resuelven racionalmente, por eso se buscan otros medios. "La educación entonces ha de verse como el gran laboratorio de la racionalidad; pero es mucho más realista si la vemos como aquel contexto en el que los jóvenes aprenden a ser razonables" (Lipman, 1997, p. 58). No solo basta con ser razonables, es necesaria una educación como un sistema que posee componentes o elementos que se interrelacionan:

Además se considera un sistema educativo complejo, y por tanto es indispensable tener presente una visión analítica, crítica, comprensiva e interdependiente de los diversos procesos educativos. Este sistema es abierto y activo, susceptible de adaptación y modificación en cada contexto, pues posee la característica de la apertura sistémica y organizacional. Es, por tanto, una posible guía para el análisis y la acción en el margen de posibilidades ofrecido a cada fenómeno educativo y formativo en concreto (Arroyave, 2002, p. 217).

El rol del maestro en el desarrollo del pensamiento complejo en el estudiante no puede limitarse a la multiplicidad de pedagogías y herramientas didácticas, necesita ser consciente de ese quehacer, porque en él permuta los paradigmas de la educación. En el estudiante hay que sembrar un sujeto interactúante con los contextos, que lea una realidad y haga una descripción de lo apreciado en ella, que exprese sus ideas realizando un ejercicio analítico y sintético para la retroalimentación de su proceso de enseñanza/aprendizaje.

El maestro, al desarrollar una visión diferente de los paradigmas, realiza una síntesis que integra, no como acumulado de realidades o disciplinas, sino como manifestación de un interés para que se dé una enseñanza a partir del trabajo colaborativo y la formación pluridisciplinar. Realizado este ejercicio el papel 
del educador va más allá de un transmisor de conocimiento; se convierte en un acompañante, un guía que fomenta el arte de pensar holísticamente en cada una de las áreas del conocimiento que acompaña. Desarrollar el pensamiento complejo en el juego filosófico es una apuesta al proceso de enseñanza/aprendizaje/evaluación. Sin embargo, ¿qué tipo de juego se pretende llevar a cabo, para poder ir construyendo una sociedad que forme seres humanos críticos, investigadores, asertivos, proactivos, participantes y creativos? Sófocles (como se citó en Paredes, 2002) nos recuerda que "el que olvidó jugar que se aparte de mi camino porque para el hombre es peligroso" (p.19) y es justo a ello a lo que pretenden responder los juegos filosóficos.

\section{Juegos fillosóficos}

¿Qué es jugar?, Según Ortega (1990) “jugar no es estudiar ni trabajar, pero jugando, el niño aprende a conocer y a comprender el mundo social que le rodea" (p. 50). Por lo que es posible afirmar que, el juego como juego es ante todo una actividad libre, y como tal, se ve en aprietos al momento de formar parte del mundo de la escuela. En el artículo juego y pedagogía Aristizábal (2001) nos dice: “el juego como creación inconsciente y no esencialmente racional, ha estado mal visto por la pedagogía tradicional, que se revela como una actividad siempre consciente y racional sobre las prácticas educativas" (p. 5). Revelándose así la tensión entre juego-pedagogía. A pesar de dicha tensión entre estas actividades, los niños aprenden jugando.

Dos ejemplos significativos pueden dar cuenta de la tensión entre juegopedagogía que se establecía anteriormente. El primero de ellos es filosofía para niños que por su compleja elaboración teórica y los materiales (novelas y manuales) utilizados para el diálogo filosófico la hacen ser más partidaria de la pedagogía tradicional, razonada y manejable. Hay que recordar que el objetivo de FpN es ayudar a desarrollar en los más pequeños destrezas dialógicas y de pensamiento, así como la capacidad de participar en una discusión democrática y productiva. Lo anterior se logra cuando los mismos niños se responsabilizan de la creación de los planes y las normas de la discusión, a la vez que participan en la práctica de la indagación filosófica; mientras que el maestro o formador sirve de facilitador en el proceso de diálogo que implica el desarrollo de pensamiento filosófico.

El segundo ejemplo tiene que ver con el enfoque denominado filosofía con niños que propone abordar la filosofía y los niños, así como el posible encuentro entre ambos. Trata de que los pequeños vivan la experiencia de la filosofía, como un movimiento del pensar que atraviesa la vida de quien la practica; por lo que conlleva algo de riesgo y ausencia de certeza. La práctica de la filosofía comporta un rumbo incierto, indeterminado, peligroso, que no promete hacer de los niños personas de tal o cual manera, sino que los prepara para un pensar diferente. En este punto $\mathrm{FcN}$ habla de poner en juego la filosofía desde el encuentro que 
los mismos pequeños tengan de ella. Una experiencia impredecible de pensamiento filosófico que se acerca más a la actividad libre del juego (Kohan, 2005).

Como se puede ver en los anteriores ejemplos, la tensión está siempre presente entre el mundo pedagógico y el juego que busca una participación más activa por parte del niño en el proceso educativo. La pregunta es ¿Qué tan grave es la tensión entre estas actividades? ¿Será posible plantear una distensión entre ambas? Tal vez, una posible respuesta por el momento sea que la práctica de la filosofía en la escuela y para la escuela debe ser pensada desde otra lógica.

Hasta el momento se sabe que el mundo del niño es lo que el adulto quiere que sea; porque este último no está preparado para reconocer al niño desde su niñez, por eso busca la forma de entenderlo como si el niño fuera un espejo suyo y ficticiamente crea el derecho de pensar qué es lo mejor para él, pero sin escucharlo, sin tomarlo enserio; porque ¿cómo podría ser tomado en serio un otro-yo diferente a mí, que juega? El adulto parece no estar de acuerdo cuando se piensa el juego como vehículo de aprendizaje y comunicación ideal para el desarrollo de la personalidad y la inteligencia del niño. Esto puede deberse en gran parte a que el juego del infante no puede ser controlado por el adulto y este último al sentirse en desventaja, lo más lógico es que quiera buscar la manera de tener nuevamente el control; pero a pesar de todo, la diferencia resiste.

Ahora la pregunta es, ¿por qué los niños muestran resistencia ante los programas educativos que se piensan para ellos? Una posible interpretación nos dice que es casi imposible que un niño aprenda si no hay juego de por medio. Buytendijk (1935) habla del juego como movimiento, espontaneidad, alegría y libre esparcimiento. Dice que el niño distingue muy bien lo que es juego de lo que no merece serlo. Tal vez a la escuela se le olvidó jugar, como dijo Sófocles, y el niño entiende el peligro que representa dicho olvido. Por eso estas reflexiones alrededor de la academia y del mundo lúdico pretenden resaltar la importancia que debería tener el juego y el niño en el ámbito educativo.

Precisamente buscándole una oportunidad al juego se pensó y se construyó una idea que sirviera de puente para unir pedagogía y mundo lúdico. Ésta propuesta es la de juegos filosóficos, donde el juego y la filosofía trabajan mancomunadamente para potencializar el pensamiento crítico y creativo en los niños, pero desde su ser-niño. Aquí el papel de la filosofía no lleva consigo un pensamiento sistemático, porque no se le quiere imponer al niño una imagen única y ordenada de la realidad; sino buscar que el juego del niño atraviese las puertas de la filosofía -como práctica- con todo lo que es él, donde su percepción individual de lo que es la realidad pueda ser escuchada y hablada a través de la acción lúdica. Porque no hay nada más concreto que el Ser jugado.

Por lo tanto, una característica del juego es la que se comprende como acción desinteresada y autotélica, Aristizábal (2001) garante de esta afirmación, sugiere que dicha acción se realice sin objetivo alguno exterior a sí misma. Esto quiere decir que el que juega lo hace por puro placer. De ahí que sí importa la forma en 
que interviene el mundo de la escuela en el proceso de juego, porque mediante este, el niño aprende pautas de comportamiento para crecer y aprender a vivir en la sociedad de una manera integral; proporcionándole interés por el conocimiento y desarrollando una actitud activa, positiva y crítica que le permitirán integrarse de manera gradual en la familia, en la escuela y en la vida.

Algunas cuestiones básicas que se deberían preguntar para adquirir conocimientos teóricos acerca del juego son: ¿Qué se entiende etimológicamente por juego? Según Paredes (2002) "el término 'juego' proviene de la raíz indoeuropea aig que significa dudar, oscilar y moverse" (p. 23). ¿Qué se entiende al momento de definir juego? Algunos teóricos dejan saber que el juego es de condición ambivalente que se resiste a una definición categórica y que su significación es polisémica, puesto que implica un amplio abanico de significados y su lectura es múltiple. Se le reconoce como versátil y elástico, escapando a una ubicación conceptual definida. En este sentido, cualquier intento por darle significado sólo será capaz de captar una parte como acercamiento parcial al fenómeno lúdico, más no a una verdad que implique su totalidad.

¿Cómo es la causalidad del juego en el niño? Se piensa que a medida que el niño crece, su mismo organismo -como necesidad psicobiológica- responde de distintas formas y utiliza distintas actividades lúcidas, es decir, que el juego evoluciona con el desarrollo integral, intelectual, afectivo, emocional y físico del niño, adecuándose a los periodos críticos de su desarrollo (personales y de entorno). El juego crece con el niño hasta su edad adulta instalándose en él hasta su vejez. Algunas características destacadas por autores como Russell, Piaget, Ortega, entre otros, muestran el juego como una actividad desinteresada y autotélica; que es libre, de acción voluntaria, porque nadie está obligado a jugar, y sólo se hace por el placer y bienestar que genera.

Lin Yutang, (como se citó en Paredes, 2002) dirá: “lo que define al juego es que uno juega sin razón, y que no debe haber razón para jugar, porque jugar en sí ya es razón suficiente. En él está el placer de la acción libre, sin trabas, con la dirección que el jugador quiera darle, que tanto se parece al arte, al impulso creador" (p. 15). Tomar el juego como una forma de aprendizaje para el adulto y de crecimiento armónico para el niño es lo que podría aportar la escuela. Nutrir las experiencias significativas de vida con la libertad para jugar, para crear, para experimentar; fortaleciendo la independencia del niño, reforzándole la seguridad y la confianza en sí mismo para que pueda afrontar el mundo externo con un pensamiento interdisciplinario.

Por eso la apuesta de juegos filosóficos como metodología de aula que se piensa tan necesaria en un mundo donde el hombre ha perdido algunos valores correspondientes a su telos fundamental (la libertad, la felicidad, el sentido, el placer, entre otros). Por medio del juego queremos rescatar al niño del mundo de los adultos, queremos escucharlos, saber cómo piensan, cómo ven el mundo, cómo lo quieren experimentar, porque si el juego pasó a ser sólo el mundo del niño, lo más justo es que la escuela reconozca que le corresponde a los 
niños decidir de qué forma quieren y desean aprender, ya que, como decía Aristóteles (1970), "todos los hombres desean por naturaleza saber" (p. 2) y el niño no es la excepción a la regla. No obstante, el mundo convencional y reglamentado del adulto mutila su curiosidad natural, porque la forma bajo la cual eligieron aprender es considerada poco seria, improductiva y está asociada a la pérdida de tiempo, con lo que terminan reduciendo el juego del niño a una mera insignificancia.

Lo que hacemos con juegos filosóficos como propuesta de una Filosofía en el aula es escuchar lo que el niño tiene por decir a partir de un juego que lo involucra con su entorno y que lo lleva a reconocer a ese otro como un yo-otro que no es él mismo. Un ejemplo teórico-práctico de la apuesta se muestra a continuación:

Tabla 1. Modelo de juego filosófico

\begin{tabular}{|l|l|l|l|}
\hline Área: & Ciencias Sociales & Fecha: & \\
\hline Actividad \#: & 1 & Nombre de la actividad: & ¿Quién soy yo? \\
\hline Temas a trabajar: & \\
\hline - Conocimiento de sí mismo \\
- La otredad y alteridad
\end{tabular}




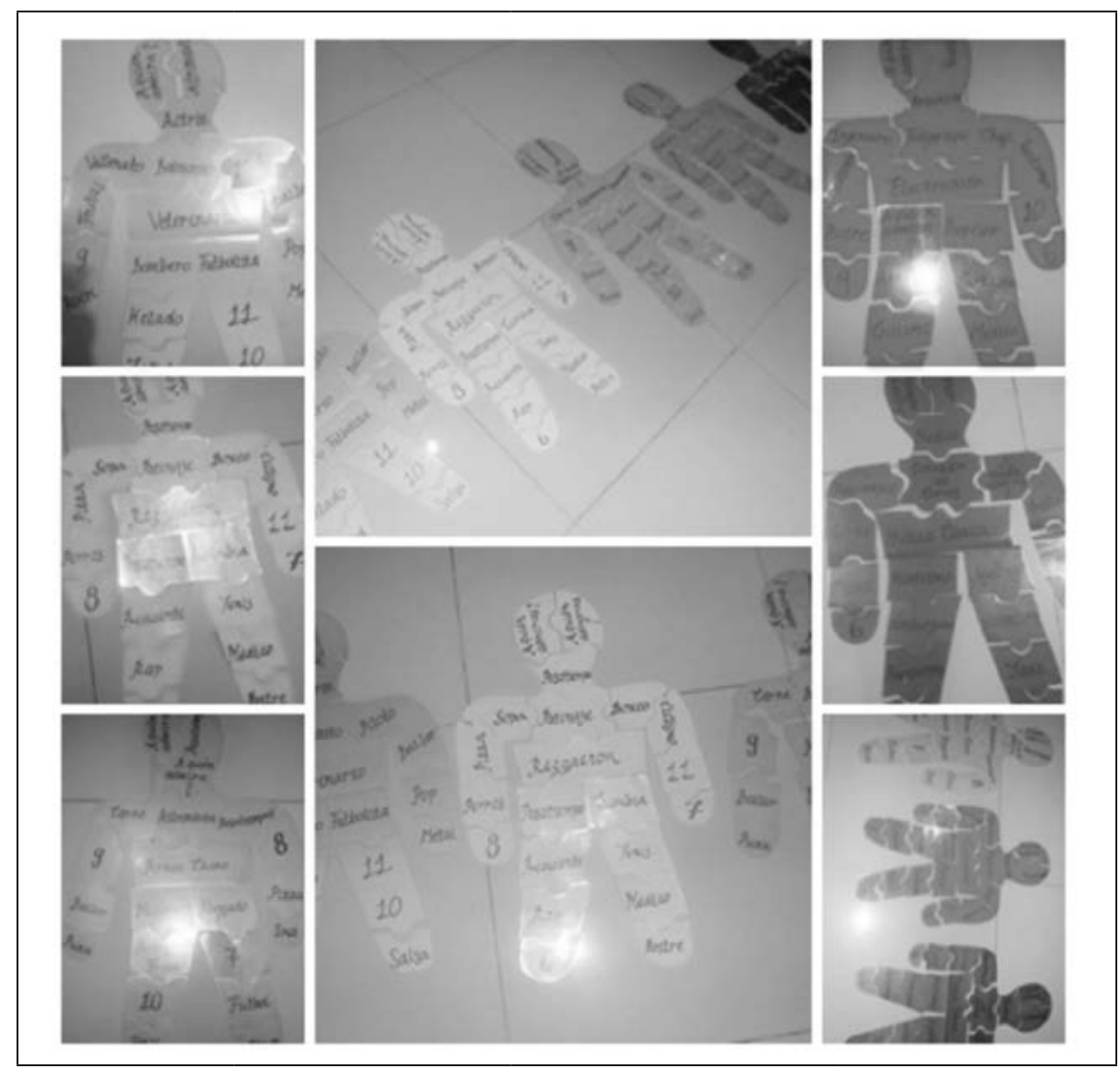

Fuente: elaboración propia.

\section{Referencias}

Aristóteles, (1970). Metafísica, Libro I. Madrid, España: Gredos.

Aristizábal, P. (2001). Juego y pedagogía. Ciencias Humanas. Recuperado de http://www.utp.edu.co/ chumanas/revistas/revistas/rev27/aristizabal.htm.

Arroyave, D. I. (2002). Manual de iniciación pedagógica al pensamiento complejo. Un encuentro entre el pensamiento moriniano y la pedagogía. Recuperado de http://online.upaep.mx/campusTest/ebooks/ManualIniciacion.pdf.

Buytendijk, F. J. (1935). El juego y su significado. Madrid, España: Occidente.

Carmona, M. (2005). Investigación ética y educación moral: el Programa de Filosofía para Niños de Matthew Lipman. Artes y Humanidades, 13(6), 
pp.101-128. Recuperado de http://www.redalyc.org/articulo.oa?id= 170121560006.

Cuno, H. (2010). Razón, racionalidad y razonabilidad, ¿Qué los identifica y diferencia? Belo Horizonte, 51(81), pp. 205-218.

Dewey, J. (1973). Mi credo pedagógico. México: FCE.

Jiménez, Y., González, M., \& Hernández, J. (2011). Propuesta de un modelo para la evaluación integral del proceso enseñanza-aprendizaje acorde con la Educación Basada en Competencias. Investigación Educativa, 13(5). Recuperado de http://www.uv.mx/cpue/num13/inves/Jimenez_modelo evaluacion.html.

Kuhn, T. (1993). Algo más sobre paradigmas. Madrid, España: FCE.

Kohan, W., \& Waskman V. (2005). Aportes para el trabajo en clase. Filosofía con niños. Buenos Aires, Argentina: Novedades Educativas.

Lipman, M. (1997). Pensamiento complejo y educación. Madrid, España: Torre.

Lipman, M. (1992). Filosofía en el aula. Madrid, España: De la Torre.

Lin, Y. (2002). Del juego. En J. Moreno (Ed.). El deporte como juego: un análisis (p. 15). Alicante, España: Miguel de Cervantes.

López, M. (2004). Notas para un replanteo del problema del sentido en el trabajo filosófico con niños. En D. Pineda., \& W. Kohan (Eds.). Filosofía para niños: debates y propuestas (p.121). Bogotá, Colombia: Beta.

Morín, E. (1983). El método II: La vida de la vida. Madrid, España: Cátedra.

Moreno, J. C. (2002). Tres teorías que dieron origen al pensamiento complejo: sistémica, cibernética e información. En M. A. Velilla (Comp.). Manual de iniciación pedagógica alpensamiento complejo (pp. 25-37). Bogotá: Instituto Colombiano para la Educación Superior-UNESCO.

Morín, E. (2003). Introducción al pensamiento complejo. Barcelona, España: Gedisa.

Noro, J. (2013). Curso de filosofía y educación para la formación y el ejercicio profesional de los docentes. Recuperado de http://es.calameo.com/books.

Ortega, R. (1990). Jugar y aprender. Sevilla, España: Diada.

Peirce, C. (1987). Obra lógico-semiótica. Madrid, España: Taurus. 
Paredes, O. (2002). Aproximación teórica a la realidad del juego. En J. Moreno (Ed.). Aprendizaje a través del juego (pp. 11-32). Málaga, España: Aljibe.

Resnick, L. (1989). Toward the Thinking Curriculum: Current Cognitive Research. Alexandria, Estados Unidos: ASCD.

Ruano, F. (1996). Racionalidad y Conciencia Trágica, la Modernidad según Max Weber. Madrid, España: Trotta S.A.

Rivera, E. (1997). De la racionalidad a la razonabilidad: ¿es posible una fundamentación epistemológica de una moral política? Hispanoamericana de Filosofía, XXIX (86), 53-81.

Salazar, B. A. (1967). Didáctica de la filosofía. Lima, Perú: Universo.

Solé, I. (1993). Estrategias de Lectura. Barcelona, Español: ICE/GRAO.

Sharp, A., \& Splitter, L. (1996). La otra educación. Buenos Aires, Argentina: Manantial.

Sófocles. (2002). Aproximación teórica a la realidad del juego. En J. Moreno (Ed.). Aprendizaje a través del juego (p. 19). Málaga, España: Aljibe.

Zuleta, E. (2010). Educación y Democracia: un campo de debate. Recuperado de http://www.omegalfa.es. 